\title{
Evaluation of Current Coil Positioning for an Enhanced Hybrid Active Space Radiation Bio-Shielding Concept
}

\author{
Jiahui Song ${ }^{1}$, Ravindra P Joshi ${ }^{2 *}$, Leroy Fung ${ }^{2}$ and Ram K Tripathi ${ }^{3}$
}

${ }^{1}$ Department of Electrical Engineering and Technology, Wentworth Institute of Technology, Boston, MA 02115, USA

${ }^{2}$ Department of Electrical \& Computer Engineering, Old Dominion University, Norfolk, VA 23529, USA

${ }^{3}$ NASA Langley Research Center, Hampton, VA 23681, USA

\begin{abstract}
Developing successful and optimal solutions to mitigating the bio-hazards of severe space radiation is critical for the success of deep-space explorations. A recent report has explored the feasibility of using a hybrid configuration that utilized both electrostatic and magnetostatic fields. Here we extend the analyses in an effort to optimize the hybrid configuration. These include changes in the radius of the current-carrying ring, and the use of multiple rings for greater bio-protection. Our simulation results show GCR proton transmission to be reduced down to 15 percent at energies around $1 \mathrm{GeV}$ for a magnetic ring with a 70 meter radius. Use of three orthogonal rings is predicted to reduce the $1 \mathrm{GeV}$ GCR proton transmission to $\sim 12$ percent, even with smaller (35 meter radius) rings.
\end{abstract}

Keywords: Radiation; Shielding; Electrostatic; Magnetostatic; Deepspace

\section{Introduction}

One of the biggest obstacles to human space exploration of the solar system is the risk posed by prolonged exposure to space radiation. Space crews traveling aboard interplanetary spacecraft will be exposed to a constant flux of Galactic Cosmic Rays (GCR), as well as intense fluxes of charged particles during Solar Particle Events (SPEs). While the particle flux for SPE falls off steeply with increasing energy (and typically has levels below $100 \mathrm{MeV}$ per nucleon), the GCR spectrum peaks around $800 \mathrm{MeV}$ per nucleon before falling off [1]. Furthermore, it is generally agreed that particles with energies around $1-2 \mathrm{GeV}$ per nucleon (before impinging on the spacecraft or any shielding material) are the most damaging to humans [2]. An additional difficulty is that crews aboard interplanetary spacecraft (such as the mission to Mars envisioned by NASA) would be exposed to the harsh radiation environment for extended periods of time. Estimated transit times for a human mission to Mars vary from 100 to 150 days each way with a possible extended duration stay on Mars estimated to be 200 days [3].

Passive shielding techniques essentially entail using solid material to create a shield which prevents particles from penetrating a given region by absorbing the energy of incident particles. Based on such conventional radiation shielding strategies, an interplanetary spacecraft would require substantial shielding if the limits for astronaut exposure are not to be exceeded [4-6]. Though other materials (including carbon or boron-nitride nanotube-based shielding) might reduce the weight somewhat, these passive shielding strategies amount to adding "dead mass" to a spacecraft which is not an economically viable solution. Furthermore, collisions of the incoming ionized particles with the passive material lead to secondary radiation, thereby introducing an additional detrimental aspect from the standpoint of health and safety.

Active shielding, on the other hand, relies on using electric [7-9], or magnetic fields [10-13] to deflect particles from a region surrounding the spacecraft. However, a different set of issues both technical and practical can then potentially arise in using such stand-alone strategies. The most serious is the safety concerns are the exceptionally large voltages ( $>1$ kilo-Volts) or large magnetic fields ( $>1$ Tesla) that would be required for shielding [14]. However, the recommended exposure limits against magnetic fields for longtime exposures has been set to
$0.4 \mathrm{~T}$ for the general public [15]. Magnetic fields can set up damaging currents within blood vessels, and lead to other adverse effects on biological tissues [16-18]. Since blood contains iron (Fe) which is a ferromagnetic substance in the form of hemoglobin, it can be affected by strong external magnetic fields.

Though the magnetic and electrostatic shielding approaches appear to have some advantages over the traditional passive shielding, the use of a single technology alone (whether electrostatic or magnetostatic) could have some serious drawbacks. For example, with electrostatic shielding along, there is the danger that high energy particles (e.g., the GCR spectra) cannot be stopped, and that even low-energy plasma may continually neutralize charges on the outermost electrostatic shields. Using static magnetic fields alone, if the coils were to be located in close proximity to the spacecraft living for better shielding, then the magnetic field-strengths necessary for protection against GCR particles would be well above $10 \mathrm{~T}[19,20]$. While the amount of electrical current needed to sustain a given magnetic dipole moment decreases with increasing coil size, the shielding capacity is also reduced significantly and almost no shielding occurs in a region near the center of the coil [20].

A combination of the electrostatic and magnetostatic shielding can present a far superior alternative, and this possibility has recently been studied [21]. The coupled, dual-approach was shown to lower the energy requirements. The basic geometry is shown in Fig. 1, consisting of six outer spheres held at a negative potential $\left(-\mathrm{V}_{\text {neg }}\right)$, six inner spheres held at a positive potential $\left(\mathrm{V}_{\mathrm{pos}}\right)$, and superconducting ring (carrying a loop current I) for providing the magnetic field. The innermost blue sphere denotes the volume (spacecraft) to be shielded. The six outer negatively charged spheres are designed to play a role in repelling the free electrons from the solar wind [7], and were used merely for

${ }^{*}$ Corresponding author: Ravindra P. Joshi, Department of Electrical \& Computer Engineering, Old Dominion University, Norfolk, VA 23529, USA, Tel: +1 757683 4827; Fax: +1 757683 3220; E-mail: rjoshi@odu.edu

Received April 02, 2013; Accepted June 24, 2013; Published June 26, 2013

Citation: Song J, Joshi RP, Fung L, Tripathi RK (2013) Evaluation of Current Coil Positioning for an Enhanced Hybrid Active Space Radiation Bio-Shielding Concept. Astrol Outreach 1: 103. doi: 10.4172/2332-2519.1000103

Copyright: ( 2013 Song J, et al. This is an open-access article distributed under the terms of the Creative Commons Attribution License, which permits unrestricted use, distribution, and reproduction in any medium, provided the original author and source are credited. 
comparing an earlier "all-sphere" design that had been reported [22]. Since negative particles typically have low energies $(<10 \mathrm{keV})$, these can be stopped by a passive shield or deflected by magnetic fields from the current-carrying ring used in our hybrid design. Finally, for completeness, it may be mentioned that setting up the electrostatic configuration might not be too arduous, especially if advances in flexible gossamer materials are employed [23]. Though this has not actually been tested or deployed, conceptually it would appear to be a plausible strategy for the electrostatic shielding component. The magnetic ring(s) that may be used as part of a hybrid active shielding approach would comprise of high Tc superconductors for low losses and high circulating currents. The low temperature of outer space would provide an ideal environment and pose no problems for the superconductors.

Here our goal is to probe the possible optimization of the hybrid structure. Specifically, potential enhancements produced by varying the size of the magnetic ring, and/or by having multiple current-carrying rings will be assessed. A Monte Carlo simulation will be used to mimic the trajectories of incoming ions, with characteristics corresponding to SPE and GCR environments taken from the literature. Finally, the possibility of using six inner positively charged spheres and magnetic rings alone, without the second set of (outer) negative spheres, will be evaluated. This would potentially hold an advantage in terms of a simpler system assembly and deployment. For such a possibility, one would need to ensure the ring(s) were positioned to completely encircle the electrostatic components. This would ensure the deflection of negative charges and the space-plasma through the Lorentz forces.

\section{Materials and Methods}

For a given potential configuration, the influence that the external fields have on incident charged particles via the collective Coulomb and Lorentz forces can lead to regions of space within which particles below some energy are unable to enter. These "forbidden" regions of space are said to be shielded from the incoming particles. The equation of motion for the incident charged particles due to a combination of electrostatic fields $\mathrm{Ex}, \mathrm{Ey}, \mathrm{Ez}$ and magnetic fields $\mathrm{Bx}, \mathrm{By}$, and $\mathrm{Bz}$ is given by:

$$
\begin{aligned}
& \mathrm{d}\left(\gamma \mathrm{m}_{i} \mathrm{v}_{x}\right) / \mathrm{dt}=\mathrm{q}\left[\mathrm{E}_{x}+\left(\mathrm{v}_{y} \mathrm{~B}_{z}-\mathrm{v}_{z} \mathrm{~B}_{y}\right)\right], \\
& \mathrm{d}\left(\gamma \mathrm{m}_{i} \mathrm{dv}_{y}\right) / \mathrm{dt}=\mathrm{q}\left[\mathrm{E}_{y}+\left(\mathrm{v}_{z} \mathrm{~B}_{x}-\mathrm{v}_{x} \mathrm{~B}_{z}\right)\right], \\
& \text { and }, \mathrm{d}\left(\gamma \mathrm{m}_{i} \mathrm{dv}_{z}\right) / \mathrm{dt}=\mathrm{q}\left[\mathrm{E}_{z}+\left(\mathrm{v}_{x} \mathrm{~B}_{y}-\mathrm{v}_{y} \mathrm{~B}_{x}\right)\right], \\
& \text { with, } \mathrm{E}_{i j}=\sum_{j} \mathrm{q}_{i} \mathrm{Q}_{j}\left(\mathrm{r}_{i}-\mathrm{r}_{j}\right) /\left[4 \pi \varepsilon_{0}\left|\mathrm{r}_{i}-\mathrm{r}_{j}\right|^{3}\right],
\end{aligned}
$$

Where $m_{i}$ is the rest mass of the $i^{\text {th }}$ particle, $r_{i}$ and $r_{j}$ are the position vectors of the incoming particle and the center of the sphere held at a surface potential $V_{j}$, respectively. Also, in the above equation, the summation is over all the charged spheres in the shielding configuration, $\gamma=\left(1-\left|\mathrm{v}_{\mathrm{i}}\right|^{2} / \mathrm{c}^{2}\right)^{1 / 2}$ is the relativistic correction factor, $c$ is the speed of light, $\varepsilon_{0}$ is the free-space permittivity, and $E_{i j}$ the electrostatic field at the location of the charge $q_{i}$. The magnetic field components $\mathrm{B}_{x}, \mathrm{~B}_{y}$ and $\mathrm{B}_{z}$ for a current carrying loop of radius "a" oriented in the $\mathrm{x}-\mathrm{y}$ plane can easily be computed from the Bio-Savart law, yielding:

$$
\begin{aligned}
& \mathrm{B}_{z}=\left[\mu_{0} \mathrm{I} /(2 \mathrm{a} \pi \mathrm{Q})\right]\left[\mathrm{E}(\mathrm{k})\left\{1-\alpha_{1}^{2}-\alpha_{2}^{2}\right\} /\left\{\mathrm{Q}-4 \alpha_{1}\right\}+\mathrm{K}(\mathrm{k})\right], \\
& \mathrm{B}_{x}=\cos (\varphi)\left[\mu_{0} \mathrm{I} \alpha_{3} /(2 \mathrm{a} \pi \mathrm{Q})\right]\left[\mathrm{E}(\mathrm{k})\left\{1-\alpha_{1}^{2}-\alpha_{2}^{2}\right\} /\left\{\mathrm{Q}-4 \alpha_{1}\right\}+\mathrm{K}(\mathrm{k})\right], \\
& \text { and } \mathrm{B}_{y}=\sin (\varphi)\left[\mu_{0} \mathrm{I} \alpha^{3} /(2 \mathrm{a} \pi \mathrm{Q})\right]\left[\mathrm{E}(\mathrm{k})\left\{1-\alpha_{1}^{2}-\alpha_{2}^{2}\right\} /\left\{\mathrm{Q}-4 \alpha_{1}\right\}+\mathrm{K}(\mathrm{k})\right],
\end{aligned}
$$

where $I$ is the current in the superconducting loop, $\mu_{0}$ is the free space permeability, $\mathrm{a}$ is the loop radius, $\mathrm{Q}=\left(1+\alpha_{1}\right)^{2}+\alpha_{2}^{2}, \alpha_{1}=\left[\left(\mathrm{x}^{2}+\mathrm{y}^{2}\right)^{1 / 2}\right] / \mathrm{a}$, $\alpha_{2}=\mathrm{z} / \mathrm{a}, \alpha_{3}=\mathrm{z} /\left[\left(\mathrm{x}^{2}+\mathrm{y}^{2}\right)^{1 / 2}\right], \mathrm{k}=\left(4 \alpha_{1} / \mathrm{Q}\right]^{1 / 2}, \varphi=\tan ^{-1}(\mathrm{y} / \mathrm{x})$, while $\mathrm{K}(\mathrm{k})$ and $\mathrm{E}(\mathrm{k})$ are the complete elliptic integrals of the first and second kind, respectively. Here for computational simplicity, the elliptic integrals were evaluated using an approximation based on the three-point Gauss quadrature [24]:

$$
\mathrm{K}(\mathrm{k})=0.512 /\left(1-0.776 \mathrm{k}^{2}\right)^{1 / 2}+0.258 /\left(1-0.987 \mathrm{k}^{2}\right)^{1 / 2}+0.8 /\left(1-0.177 \mathrm{k}^{2}\right)^{1 / 2},
$$$$
\text { and, } \mathrm{E}(\mathrm{k})=0.232\left(1-0.993 \mathrm{k}^{2}\right)^{1 / 2}+0.707\left(1-0.115 \mathrm{k}^{2}\right)^{1 / 2}+0.632\left(1-0.751 \mathrm{k}^{2}\right)^{1 / 2}
$$

Here, calculations for charged particle penetration have been performed using Monte Carlo simulations to obtain trajectories in the presence of the specified dual electrostatic-magnetostatic configuration of figure 1. The kinetic-based, Monte Carlo numerical simulations followed the trajectories of 10,000 particles. These particles were taken to be injected inwards at random angles from a spherical simulation boundary of radius 500 meters. Trajectories of each ionized particle were computed based on the relativistic equations (2a-2c) of motion. By tracking the trajectories of all the 10,000 simulated particles, this process naturally allowed for the evaluation of the fraction penetrating the central region, those completely deflected by the active shielding arrangement, and the fraction incident onto the spheres or the ring.

In order to accurately gauge space radiation responses to the shielding system, Monte Carlo simulations were performed for incoming particles of a fixed energy. For clarity, transmission of particles with specific energies (i.e., mono-energetic incident charged particles) was evaluated. For an overall picture, the transmission weighted over the spectra for Solar Particle Events (SPEs) and Galactic Cosmic Rays (GCRs) could easily be folded in. For example, the GCR model spectra [25] could be used to gauge the impact of the hybrid shielding from all of the high energy particles.

\section{Results}

An important task in devising the hybrid shielding system is to determine the radius and current through the ring for optimal deflection of the incident ions. A larger current would produce a higher magnetic field, and be a more effective active shield. However, caution has to be exercised to ensure that the magnetic field values within the spherical shielded region produced by the ring are below the safety threshold of $0.4 \mathrm{~T}$ [15]. As will be shown in the numerical results, a

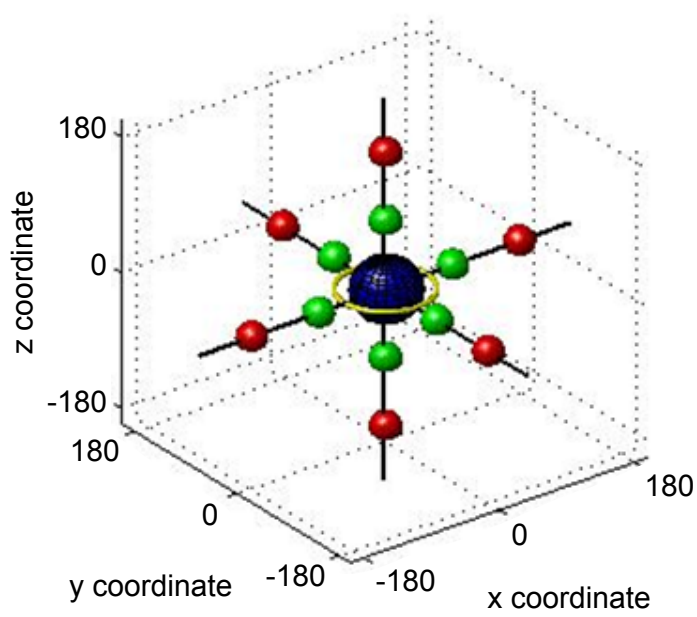

Figure 1 (color): Geometry considered for a dual electrostatic-magnetostatic shielding configuration. The radii of the outer (negatively charged) and inner (positively charged) spheres were taken to be 20 and 10 meters and located at mean distances of 160 and 50 meters, respectively, from the center. An additional current-carrying ring shown produces magnetic fields. The radius and current of this ring was taken to be a variable parameter. 
larger ring radius helps keep the peak location of the magnetic field further away from the central protected zone. Under this situation, the current for a larger ring can then be increased to produce stronger magnetic fields further away, while still ensuring the peak value within the inner spherical protected zone (assumed to be a sphere of radius 20 $\mathrm{m})$, is about $0.4 \mathrm{~T}$.

Figure 2 shows the maximum values of the total magnetic field [= $\left.\left(B_{x}^{2}+B_{y}^{2}+B_{z}^{2}\right)^{1 / 2}\right]$ as a function of the ring current. These maximum magnetic field values were obtained both within the spherical protected zone of radius 20 meter, and for the overall simulated space. The ring radius for this calculation was set at 25 meters. The result shows that while a much higher magnetic field can exist (near the current-carrying ring for example), values within the inner protected zone as shown in figure $2 \mathrm{~b}$ can be significantly lower. For a larger ring radius, the permissible current could be higher while still conforming to the 0.4 Tesla thresholds within the inner $20 \mathrm{~m}$ zone.

Maximum magnetic field intensities due to a $25 \mathrm{~m}$ ring as a function of its loop current. (a) Peak value within a 50 meter region, and (b) those within a $20 \mathrm{~m}$ inner protected zone figure 2 .

(a)

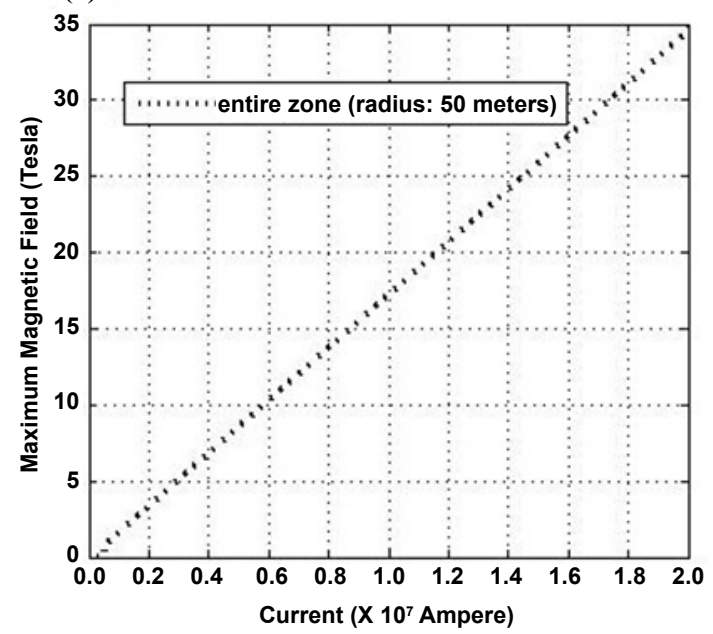

(b)

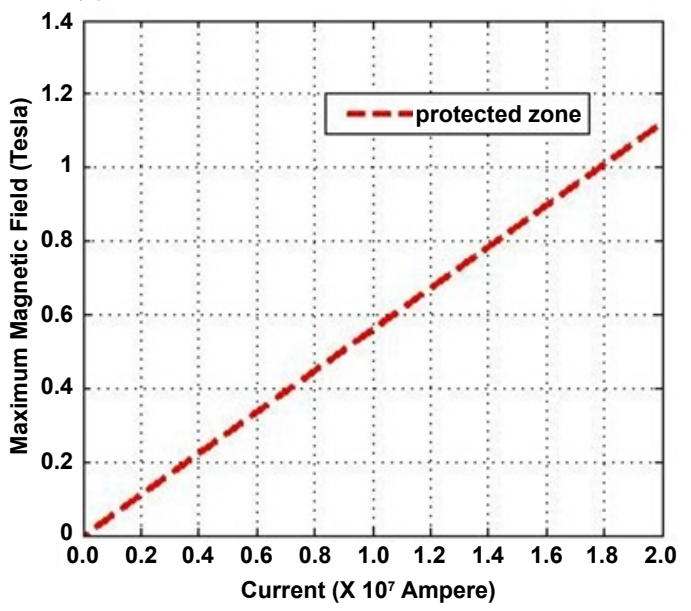

Figure 2 (color online only): Maximum magnetic field intensities due to a $25 \mathrm{~m}$ ring as a function of its loop current. (a) Peak value within a 50 meter region, and (b) those within a $20 \mathrm{~m}$ inner protected zone.
The possibility of creating fairly large magnetic fields, while ensuring values below the 0.4 Tesla thresholds has thus been demonstrated. Next, numerical simulations were carried out to ascertain the potential for successful yet safe active shielding for the configuration of Figure 1 . The system included the twelve charged spheres and one current-carrying ring. The six outer negatively charged spheres were taken to be at a -100 MV potential, while the six inner positively charged spheres were each set at $100 \mathrm{MV}$. The radii of the outer and inner sets of spheres were 20 and 10 meters, respectively, and located at mean distances of 160 and 50 meters, respectively, from the center. These numbers generally reflect the values reported in Ref. 22 for an all-electrostatic configuration. The inner zone to be protected was taken to be a spherical region of radius 20 meters.

Predictions from numerical simulations for GCR proton transmission probability as a function of the incident energies for three different magnetic ring sizes are shown in figure 3 for the 12-sphere, 1 -ring configuration of figure 1 . The currents were adjusted for each of the three ring sizes (corresponding to three different simulation cases) to ensure the peak magnetic intensities within the inner 20 meter zone remained below 0.4 Tesla. Results of figure 3 show a substantial reduction in GCR transmission for the largest 70 meter ring. In fact, for the critical energies of the GCR spectra around $1 \mathrm{GeV}$ per nucleon, only about 15 percent of the incident flux is predicted to breach the spacecraft. This is a substantial reduction, as compared for example, to an all-electrostatic configuration reported a few years ago [22]. In that all-electrostatic, 12-sphere configuration, transmission probabilities were reported to be as high as 46 percent, and that too after a much higher $300 \mathrm{MV}$ electrostatic bias.

Simulation results of energy dependent probability of protons to penetrate through a 20 meter spherical zone for the 1-ring and 12 -sphere shielding configuration. Three different current-carrying ring sizes were used (Figure 3 ).

Next, a comparison of the energy dependent proton penetration between the 12-sphere and 6-sphere configurations was probed. Such a comparison was guided in part by the need to reduce the shielding system complexity down to six electrostatic spheres. For an alternative, 6-sphere configuration, the six outer negatively charged spheres were

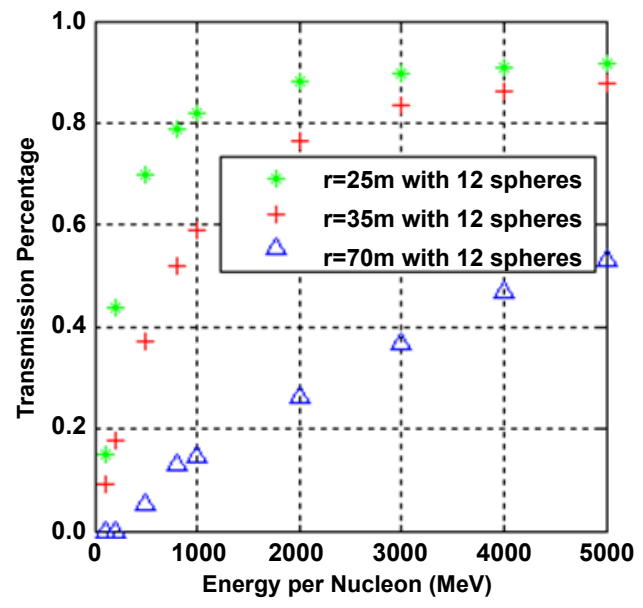

Figure 3 (color online only): Simulation results of energy dependent probability of protons to penetrate through a 20 meter spherical zone for the 1-ring and 12-sphere shielding configuration. Three different current-carrying ring sizes were used. 
removed. This is justifiable given that negative particles typically have low energies $(<10 \mathrm{keV})$, and can be stopped by a passive shield or deflected by magnetic fields from the current-carrying ring used in our hybrid design. Simulation results are given in figure 4 for three different ring radii of 25 meters, 35 meters and 70 meters. Differences between the 12- and 6-sphere cases at each of the magnetic ring sizes are not very significant. In all cases, the transmission for the 12-sphere configuration is marginally larger as compared to its six-sphere counterpart. Physically, this is easily understood from the added acceleration provided to the incoming protons by the six outermost negatively charged spheres. This clearly points to the possibility of removing the six outer spheres and deploying the more compact and simpler 6-sphere, 1-ring shielding combination. It may be mentioned for completeness, that the presence of the magnetic field would deflect electrons and other negative plasma ions. Hence, unlike an allelectrostatic system [22], an outer negatively charged layer might not be necessary given the inclusion of magnetostatic fields in this hybrid configuration.

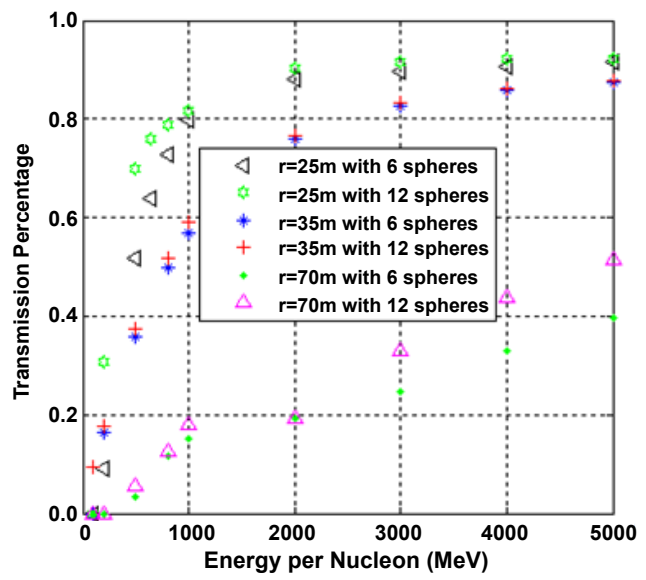

Figure 4 (color online only): Simulation results comparing the energy dependent transmission of protons through a 20 meter spherical zone for the 12 -sphere and 6 -sphere shielding configurations. A single magnetic ring was also used for each case.

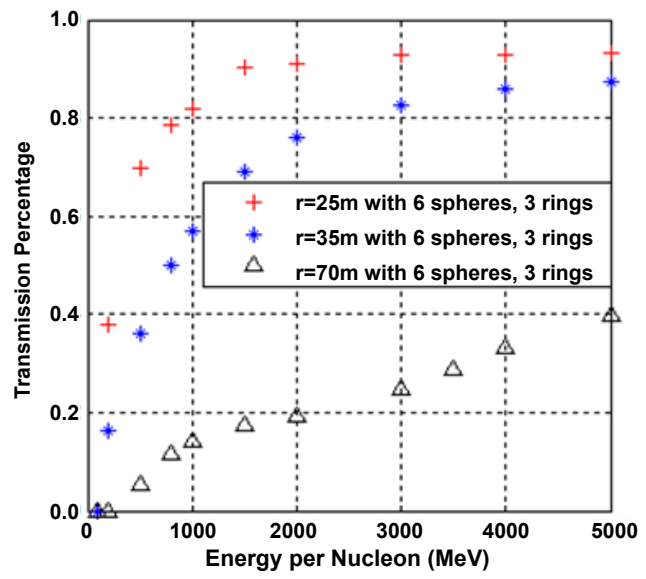

Figure 5 (color online only): Results showing the predicted energy dependent proton penetration for shielding configurations consisting of six positively charged spheres and three mutually orthogonal magnetic rings. Three different ring radii of $25 \mathrm{~m}, 35 \mathrm{~m}$ and $70 \mathrm{~m}$ were chosen.
Simulation results comparing the energy dependent transmission of protons through a 20 meter spherical zone for the 12-sphere and 6-sphere shielding configurations. A single magnetic ring was also used for each case figure 4 .

Since the magnetic field plays an important role in the shielding, especially at the higher energies, it is instructive to evaluate the potential advantage in having multiple current-carrying rings. Towards this goal then, the 1-ring case was extended to a three-ring configuration by placing mutually orthogonal rings in the $x-y$ plane, the $y-z$ and $z-x$ planes. Results for the energy-dependent proton penetration obtained for a 6 positive sphere and 3-ring configuration are shown in figure 5 . Three different ring radii of $25 \mathrm{~m}, 35 \mathrm{~m}$ and $70 \mathrm{~m}$ were again chosen.

Results showing the predicted energy dependent proton penetration for shielding configurations consisting of six positively charged spheres and three mutually orthogonal magnetic rings. Three different ring radii of $25 \mathrm{~m}, 35 \mathrm{~m}$ and $70 \mathrm{~m}$ were chosen figure 5 .

Inclusion of three rings rather than just one-ring is predicted to improve the shielding and lower the proton GCR penetration fraction. This aspect can perhaps be better seen with more clarity through figure 6, which compares the 1-ring and 3-ring cases for a fixed $35 \mathrm{~m}$ radius. For example, at the $1 \mathrm{GeV}$ energy for the protons, while the 1-ring, 6-sphere configuration is predicted to roughly allow a fifty eight percent transmission. However, the 3-ring, 6-sphere configuration has a much lower penetration level of only about fifteen percent.

Comparing results for the proton transmission fraction between a 1-ring and a 3-ring magnetic configuration. Six positively charged spheres were used, with the current-carrying rings at a fixed radius of $35 \mathrm{~m}$, in all cases figure 6.

\section{Summarizing Conclusion}

In conclusion, we have conducted detailed numerical studies to evaluate the potential for a hybrid system for active bio-shielding against space radiation. Most previous approaches have been based on a single concept alone, such as the magnetic, electrostatic or plasma shielding. However, all of the conventional approaches (including passive shielding) have potential problems. The hybrid configuration included a set of electrostatic spheres as was proposed recently, and combined one or multiple current-carrying rings to generate additional

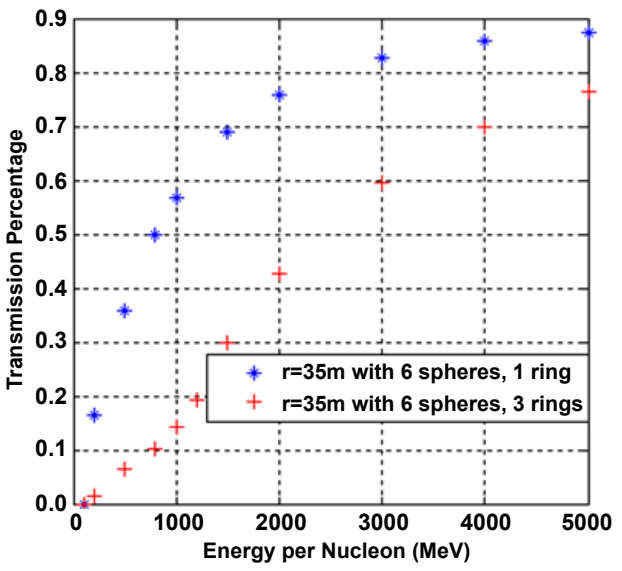

Figure 6 (color online only): Comparing results for the proton transmission fraction between a 1-ring and a 3-ring magnetic configuration. Six positively charged spheres were used, with the current-carrying rings at a fixed radius of $35 \mathrm{~m}$, in all cases. 
Citation: Song J, Joshi RP, Fung L, Tripathi RK (2013) Evaluation of Current Coil Positioning for an Enhanced Hybrid Active Space Radiation BioShielding Concept. Astrol Outreach 1: 103. doi: 10.4172/2332-2519.1000103

magnetic fields. The main advantages of this system were: (i) much lower magnetic fields that could be below the thresholds set for health and safety for long-term exposures, (ii) a much better shielding and repulsion of incident ions especially the high energy GCR spectra which has always been of critical importance, and (iii) the possibility to reduce the electrostatic voltage values over an all-electric [22] configuration.

Our results showed significant reductions in the transmission for the dual shielding system. Larger radius rings were shown to provide better radiation protection, and this aspect was further improved by employing multiple rings. Specifically, it has been shown that transmission for GCR protons can be as low as 15 percent at energies around $1 \mathrm{GeV}$ for a magnetic ring with a 70 meter radius. Use of three orthogonal rings is predicted to reduce the $1 \mathrm{GeV}$ GCR proton transmissions to $\sim 15$ percent for smaller 35 meter rings. For completeness, it must be mentioned that our study represents a simple first-step evaluation. Improvements are certainly possible by choosing other novel structures (such as charged toroidal rings instead of the spheres) or meshed designs to reduce weight and costs while still achieving the requisite active shielding. More interestingly, the dimensions and aspect ratio of the spheres (or tori) and/or rings could be altered to achieve an even higher degree of radiation protection.

\section{Acknowledgement}

This work was supported in part by NASA Innovative Advanced Concept (NIAC) Program. The team at Old Dominion University (RPJ and LF) acknowledges the NASA Langley Research Center for partial support through grant No. NNX11AG71G.

\section{References}

1. Yao WM (2006) Review of Particle Physics. J. Phys. G: Nucl. Part. Phys. 33: 245-251.

2. Schimmerling W, Cucinotta FA (2006) Dose and dose rate effectiveness of space radiation. Radiation Protection Dosimetry 122: 349-353.

3. Hoffman SJ, Kaplan DI (1997) Human Exploration of Mars: The reference mission of the NASA mars exploration study team. NASA Johnson Space Center, Houston, TX, NASA Special Publication 6107.

4. Wilson JW, Cucinotta FA, Kim MH, Schimmerling W (2001) Optimized shielding for space radiation protection. Phys. Med.17: 67-71.

5. Cucinotta FA, Kim MHY, Ren L (2006) Evaluating shielding effectiveness for reducing space radiation cancer risks. Radiation Measurements 41: 11731185.

6. Cucinotta FA, Kim MHY, Chappell LJ (2010) Space radiation cancer risk projections and uncertainties-2010. NASA/TP-2011-216155: 1-136.

7. Townsend LW (1984) Galactic heavy-ion shielding using electrostatic fields NASA Technical Memorandum 86255.

8. Sussingham JC, Watkins SA, Cocks FH (1999) Forty years of development of active systems for radiation protection of spacecraft. J. Astronaut. Sci. 47 $165-175$.
9. Smith J.G, Smith T, Williams M, Youngquist R, Mendell W (2006) Potential polymeric sphere construction materials for a spacecraft electrostatic shield NASA/TM-2006-214302. Hampton, VA, Langley Research Center.

10. Adams JH, Hathaway DH, Gregory JC, Grugel RN, Parnell TA, et al.(2005) Revolutionary concepts of radiation shielding for human exploration of space. NASA TM 213688

11. Cocks FH (1991) A Deployable High Temperature Superconducting Coil (DHTSC): A novel concept for producing magnetic shields against both solar flare and galactic radiation during manned interplanetary missions. J. British Interplanetary Soc. 44: 99-102.

12. Rossi L, Sorbi M, Spillantini PA (2004) Superconducting magnetic lens fo solar rays protection in manned interplanetary missions. IEEE Trans. Appl. Supercond. 14: 1696-1699.

13. Spillantini $P$ (2010) Active shielding for long duration interplanetary manned missions. Advances in Space Research 45: 900-916.

14. Townsend LW (2000) Overview of active methods for shielding spacecraft from energetic space radiation, 11th Annual NASA Space Radiation Health Investigators' Workshop, Arona, Italy.

15. (2009) International Commission on Non-lonizing Radiation Protection Guidelines on Limits of Exposure to Static Magnetic Fields, Health Physics 96: 504-514.

16. Kinouchi $Y$, Yamaguchi $H$, Tenforde TS (1996) Theoretical analysis of magnetic field interactions with aortic blood flow. Bioelectromagnetics 17: 21-32.

17. Kawakubo T, Yamauchi K, Kobayashi T (1999) Effects of magnetic field on metabolic action in the peripheral tissue. Journal of Applied Physics, Part 2 Letters 38: L1201-L1203.

18. Nittby H, Grafstrom G, Eberhardt JL, Malmgren L, Brun A, et al.(2008) Radiofrequency and extremely low-frequency electromagnetic field effects on the blood-brain barrier. Electromagn Bio Med.27: 103-126.

19. Shepherd SG, Shepherd JPG (2009) Toroidal magnetic spacecraft shield used to deflect energetic charged particles. Journal of Spacecraft and Rockets 46 177-184.

20. Shepherd SG, Kress BT (2007) Stormer Theory applied to magnetic spacecraft shielding. Space Weather 5: S04001 (1-6).

21. Joshi RP, Quu H, Tripathi RK (2013) Evaluation of a combined electrostatic and magnetostatic configuration for active space-radiation shielding. Advances in Space Research 51: 1784-1791.

22. Tripathi RK, Wilson JW, Youngquist RC (2008) Electrostatic space radiation shielding. Advances in Space Research 42: 1043-1049.

23. Stiles LA, Schaub H, Maute KK, Moorer DF (2013) Electrostatically inflated gossamer space structure voltage requirements due to orbital perturbations. Acta Astronautica 84: 109-121.

24. Vatankhah AR (2011) Approximate solutions to complete elliptic integrals for practical use in water engineering. J. Hydrol. Eng16: 942-945

25. O'Neill PM (2010) Badhwar-O'Neill 2010 galactic cosmic ray flux modelRevised. IEEE Transactions on Nuclear Science 57: 3148-3153. 